\title{
IDENTIFICATION OF DRIVERS DROWSINESS BASED ON FEATURES EXTRACTED FROM EEG SIGNAL USING SVM CLASSIFIER
}

M.Thilagaraj

Department of Electronics and Instrumentation Engineering Karpagam College of Engineering

Coimbatore, Tamilnadu, (India).

E-mail:m.thilagaraj@gmail.com

ORCID: https://orcid.org/0000-0002-7729-3273

M. Pallikonda Rajasekaran

Department of Electronics and Communication Engineering, Kalasalingam Academy of Research and

Education Krishnankoil, Tamilnadu, (India).

E-mail:m.p.raja@klu.ac.in

ORCID: https://orcid.org/0000-0001-6492-4512

U. Ramani

Department of Electrical and Electronics Engineering, K. Ramakrishnan College of Engineering Trichy, Tamilnadu, (India).

E-mail: ramani.eee@krce.ac.in

ORCID: https://orcid.org/0000-0003-3820-3607

Recepción: 28/11/2019 Aceptación: 16/09/2020 Publicación: 30/11/2021

\section{Gitación sugerida:}

Thilagaraj, M., Rajasekaran, M. P., y Ramani, U. (2021). Identification of drivers drowsinessbased on features extracted from EEG signal using SVM classifier. 3C Tecnología. Glosas de innovación aplicadas a la pyme, Edición Especial, (noviembre, 2021), 579-595. https://doi.org/10.17993/3ctecno.2021. specialissue8.579-595 


\section{ABSTRACT}

Electroencephalogram (EEG) is a recording machine used for storing the electrical movement of the brain. The brain waves are produced by passing electric current through the brain and that is being recorded by the Electroencephalogram. After taking the EEG signal the process of removing the noise and low-quality signal is carried out by using the Butterworth filter and that process is known as Preprocessing. Then the signal is segmented with the help of Discrete Wavelet Transform (DWT) so that the signals are segmented into five primary frequency bands (delta, theta, alpha, beta, and gamma). Finally, the EEG signals were classified based on the statistical features obtained from the different segments of the EEG signals using Support Vector Machine Classifier. SVM maps input vector to a high dimensional space where a finest hyper plane is developed. Among the numerous hyper planes accessible, there is only one hyper plane that amplifies the separation among itself and the closest information vectors of every class. The identification of the fatigue based on the features extracted using SVM is more efficient compared to the other feature extraction methods employed for the analysis of the signals.

\section{KEYWORDS}

ElectroEncephaloGram (EEG), Discrete Wavelet Transform (DWT), Statistical Features, SVM Classifier. 


\section{INTRODUCTION}

Drowsiness is the intermediate state among awaken and sleep. Many factors can cause tiredness or fatigue in driving as well as long driving hours, lack of sleep, take more medicines, eating of alcohol, and some early morning drive, mid-afternoon hours, driving at midnight, and particularly in a monotonous driving environmental setup. Driving under the influence of drowsiness will cause: Reduction in the level of concentration and reduction in the ability of a person to take decision quickly.

According to the Nationwide Road travel protection management (NHTPM), about $1,00,000$ crashes are the directly outcome of driver sleepiness each year. This is the basis why more and more researches are going on in this field. So to avoid such accidents this project has been established.

The drowsiness had been diagnosed on the premise of (Electroencephalogram) EEG, (Electrooculography) EOG, Galvanic Skin Response (GSR), coronary heart charge and pulse price and so forth, these are some of the physiological measurements

For identifying drowsiness many strategies have been used typically for the process of segmentation, tiny Time Fourier Transform (STFT) is used. Wigner-Ville Distribution (WVD) is employed to extract features from the EEG indicators. The statistical features set is being decomposed into small and finite range of intrinsic mode function by means of empirical mode decomposition technique. In the existing works benchmark datasets is used. Characteristic extraction and function class are the two main modules of biomedical signal. For studying non stationary signals time frequency illustration is used. TFRS are spoken to by method for either adequacy or vitality thickness throughout the years and recurrence.

This paper gives a brand-new approach primarily based at the aggregate of time-frequency picture and DWT the SVM to categorize the EEG signal for fatigue detection. The EEG indicators are to start with training and labeling the indicators by means of extracting features from the segmented components. Then EEG signals are segmented via employing DWT. The statistical features had been extracted from the segments. The evaluation parameters include variance, skewness, entropy and kurtosis of the segmented signals. The extracted capabilities have been after that classified using SVM Classifier. The overall 
presentation of the process is estimated through figuring exactness, and explicitness of the classifier. Right here each nonlinear and non-stationary signal are evaluated by using DWT. As a method to break down markers is posteriori and grants the mining of the inward sizes of each sign, so it has a first-rate benefit in EEG signal processing. It affords an automatic popularity of fatigue based at the time-frequency image the usage of DWT and SVM. The best capabilities of alpha, beta, gamma, theta, and delta waves are fed into the sample recognition tool for category of fatigue and non-fatigue EEG indicators.

\section{MATERIALS AND METHODS}

\subsection{RELATED WORKS}

Chen et al. (2015) proposed regular detection of awareness or sluggishness from physiological sign utilizing wavelet based nonlinear highlights and artificial intelligence physiological indicator such as electroencephalogram and electrooculography recording is much necessary non-invasive procedures of detecting someone's awareness/tiredness. We recommend a gadget for drowsiness detection the usage of physiological signals that gift 4 benefits: (1) disintegrating EEG signals into wavelet sub-groups to extract extra glaring information past uncooked signal (Chen et al., 2015), (2) extraction and combination of nonlinear features from EEG sub-bands, (3) combination the facts from EEG's and eyelid actions, (4) utilizing proficient very learning machine for notoriety order (Zhang, Wang, \& $\mathrm{Fu}, 2014)$. The preliminary outcomes demonstrate that the proposed system accomplishes not handiest a high recognition exactness but rather additionally a completely quick calculation speed (Bajaj \& Pachori, 2012).

Zhang et al. (2014) proposed automatic finding of Driver tiredness Based on Entropy and Complexity Measures. In this research work offers an actual-time approach based totally on numerous entropy and intricacy measures for recognition and identification of using exhaustion from recorded biological signal indicators (Kar, Bhagat, \& Routray, 2010; Picot, Charbonnier, \& Caplier, 2012). The entropy-based totally capabilities, particularly, the Wavelet Entropy (WE), the peak-to-top fee of apen (pp-apen), and the peak-to-height cost of pattern entropy (pp-sampen), were extricated from the collected alarms to assess the driving exhaustion levels (Kumar, Raju, \& Kumar, 2012; Picot, Charbonnier, \& Caplier, 
2011). The actual-time capabilities received via we, pp-apen, and pp-sampan with sliding window have been applied to synthetic neural community for education and trying out the machine, which gives 4 conditions for the fatigue stage of the patients, specifically, normal country, mellow weakness, emotional episode, and over the top weariness. Then, the motive force fatigue stage may be determined in actual time (Majumdar, 2011; Mardi, Ashtiani, \& Mikaili, 2011).

\subsection{METHODOLOGY}

This process includes five important modules each of them have a unique processing capability.

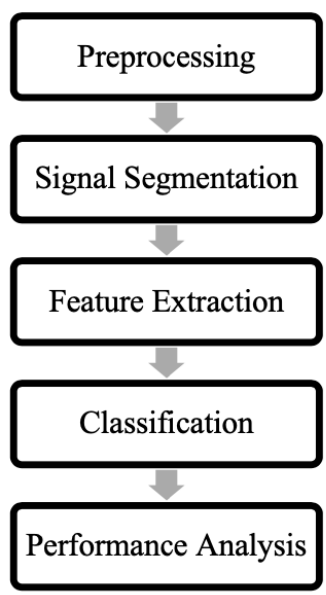

Figure1. Block diagram.

Source: own elaboration. 
The flow diagram of the module is mentioned below as follows:

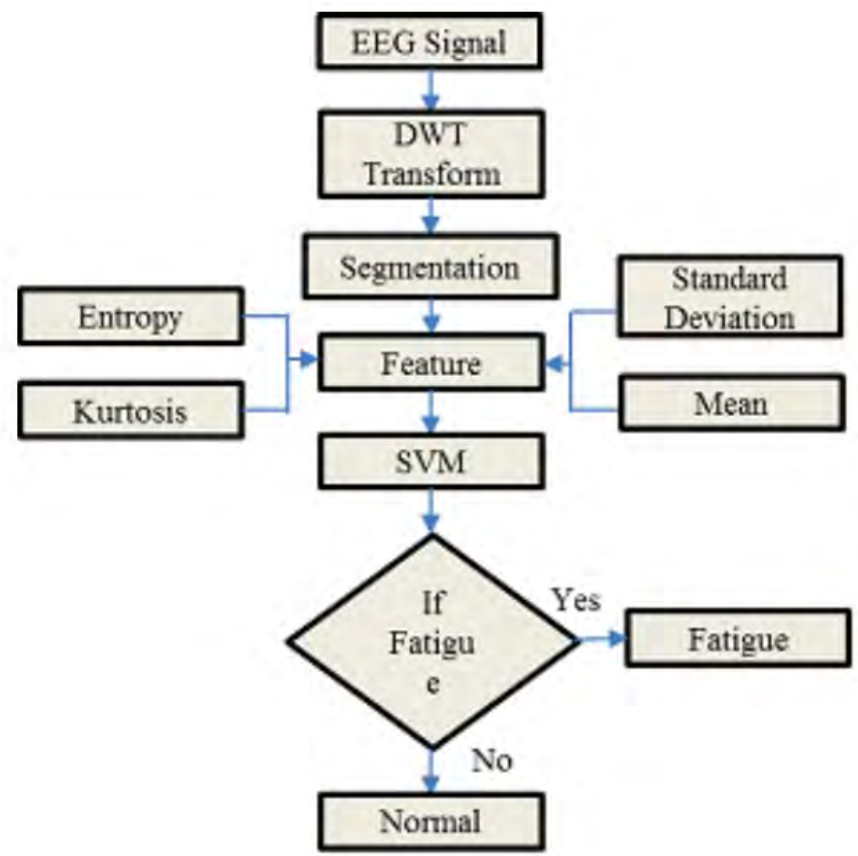

Figure 2. Flow diagram of modules.

Source: own elaboration.

\subsection{PREPROCESSING}

Preprocessing is carried out in EEG signal, why because EEG signals are not constant in nature (i.e) different frequency workings are existed in different interval of time. The input signal needs to be preprocessed before going to process the signal. To remove extracting time, unwanted noise, frequency and TF/TS domain features from the multi-channel EEG the process of preprocessing is carried out, with this technique systole, noise, low quality signal will be removed. EEG signals are preprocessed using Butterworth filter. Here Butterworth is used as both low pass and high pass filter. Before preprocessing DC, Drift Elimination is carried out for removing drift.

Butterworth filter: Butterworth filter is an ideal filter. The definition of ideal filter is that the filter that not only completely reject the unwanted frequencies but should maintain a uniform sensitivity over the wanted frequencies such a filter cannot be obtained but butterwort filter showed that by increasing the number of filter elements. Butterworth 
filter can able to adjust the component values of the filter. That his basic low pass filter functionality could be modified to give high pass, band stop, band pass functionality. Butterworth filter has maximally flat frequency response (i.e.) in the pass band it has no ripple and in the stop band it rolls off towards zero. Compared to Chebyshev and Elliptic filter, more linear phase response is achieved by the butter worth filter in the pass band.

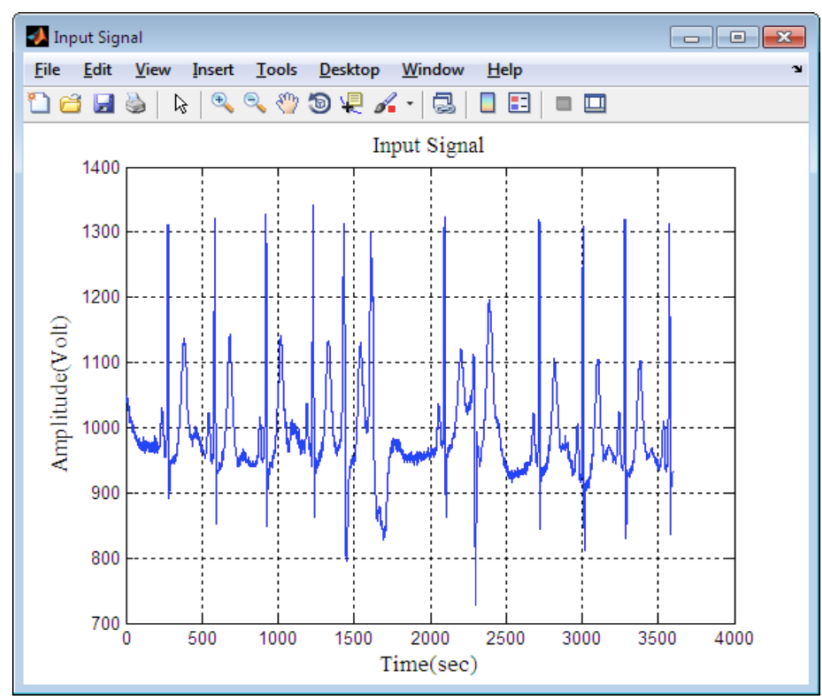

(a)

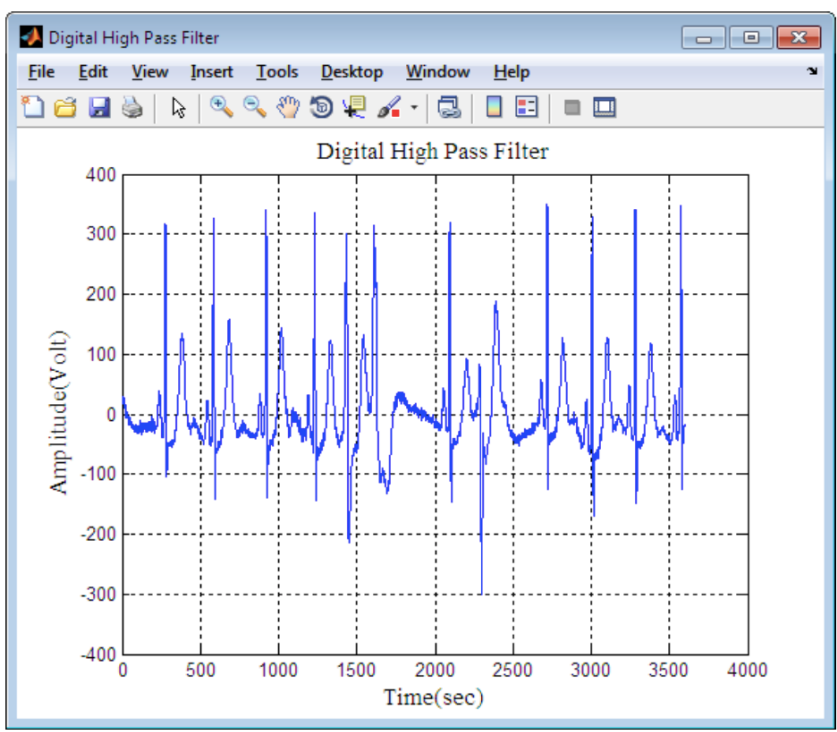

(b) 


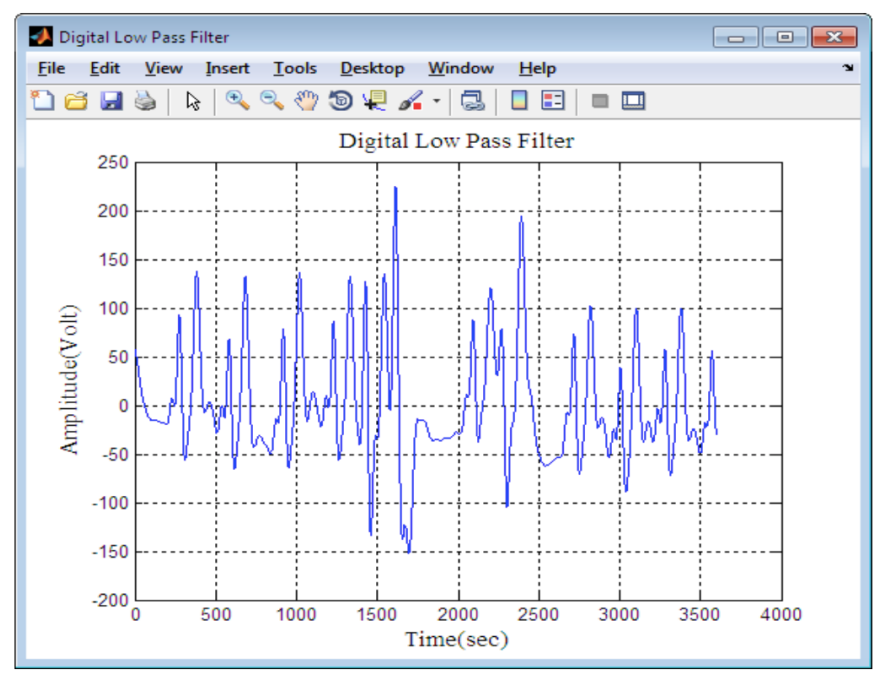

(c)

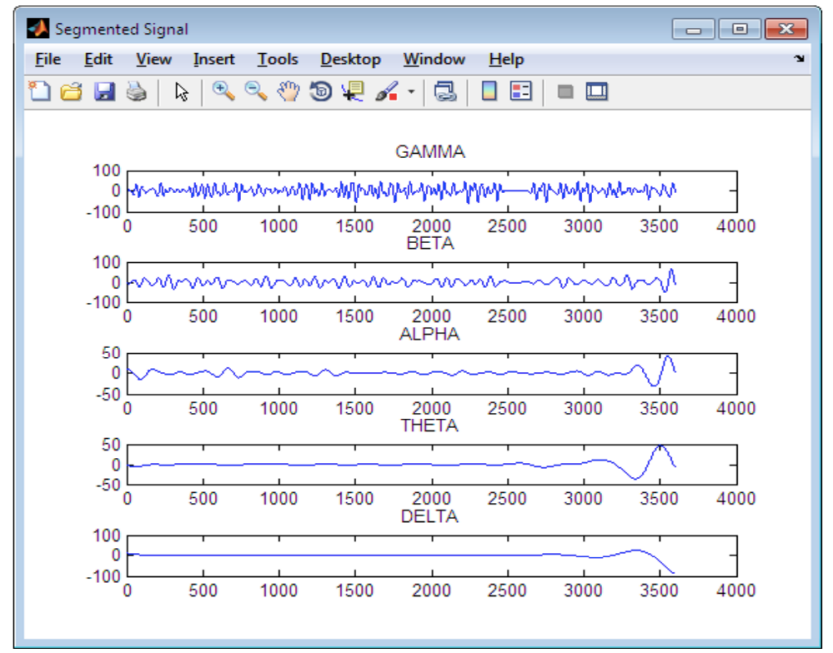

(d)

Figure 3. (a) Input Signal, (b) DC Drift Elimination, (c) Preprocessed signal using HPF, (d) Preprocessed signal using LPF.

Source: own elaboration.

\subsection{SIGNAL SEGMENTATION}

On the basis of frequency-bands of the rhythms Segmentation has been employed. EEG signal can be taken into consideration as a superposition of various structure occurring 
on distinct time scales at different time. There are two types of segmentation, the signal segments to equal part are the first type and is called constant segmentation. The advantage of this method is that it is very simple to process, the disadvantage is bad accuracy. In the $2 \mathrm{~d}$ method for segmentation of non-stationary signal is adaptive segmentation which means the signal segments robotically to wise components with equal residences.

Segmentation procedure segments the signal into five primary frequency band they're delta, theta, alpha, beta, gamma. The range of every frequency are delta ranges from $0.5 \mathrm{~Hz}$ to $4 \mathrm{~Hz}$, theta ranges from $4 \mathrm{~Hz}$ to $8 \mathrm{~Hz}$, alpha ranges from $8 \mathrm{~Hz}$ to $12 \mathrm{~Hz}$ and beta ranges from $12 \mathrm{~Hz}$ to $30 \mathrm{~Hz}$ Gamma ranges from greater than $30 \mathrm{~Hz}$

- Here Alpha, Beta sub bands are responsible for drowsiness.

- When a person is closing his eye or resting means, predominant of alpha activity is carried out.

- Transition from unsleeping to sleep nation alpha waves decreases at the same time as theta waves increases.

- Beta waves are excessive whilst someone is taking capsules.

The process of Segmentation is carried out using Discrete Wavelet Transform (DWT).

Discrete Wavelet Transform: Intrinsic multi-goals nature, wavelet-coding plans for application where versatility and acceptable deprivation are important this is why we have chosen separate Wavelet Transform. Wavelet change deteriorates a sign into a lot of premise capacities. These premise capacities are called wavelets. Separate Wavelet Transforms (SWT), which transforms a discrete time signal to a discrete wavelet symbol.

Basically, the medical images need more accuracy without loss of sequence. The Separate Wavelet Transform (SWT) was based on time-scale representation, which provides capable multi-resolution.

It has been analyzed that the Separate Wavelet Transform (SWT) operates at a maximum clock frequency of 99.197 $\mathrm{MHz}$ respectively. 


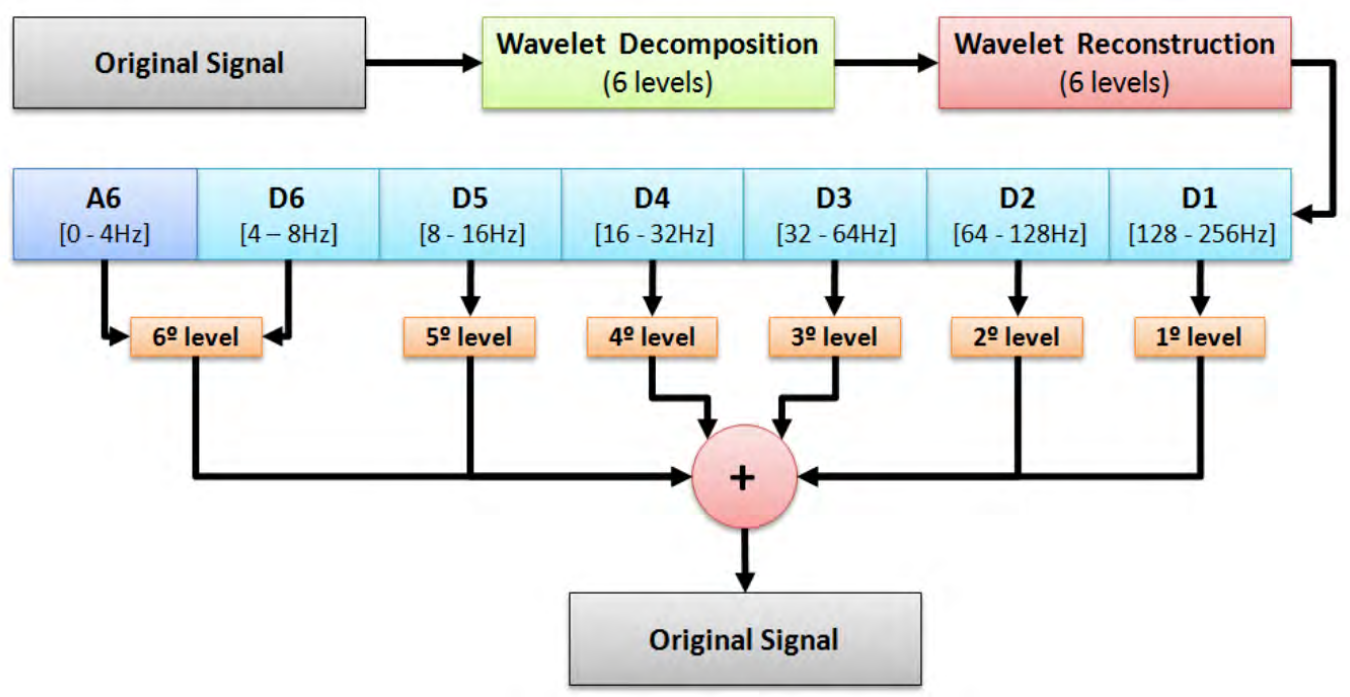

Figure 4. Discrete Wavelet Transform.

Source: own elaboration.

DWT offers end result a lot sharper than any of the conventional analysis method in the time-frequency domain.

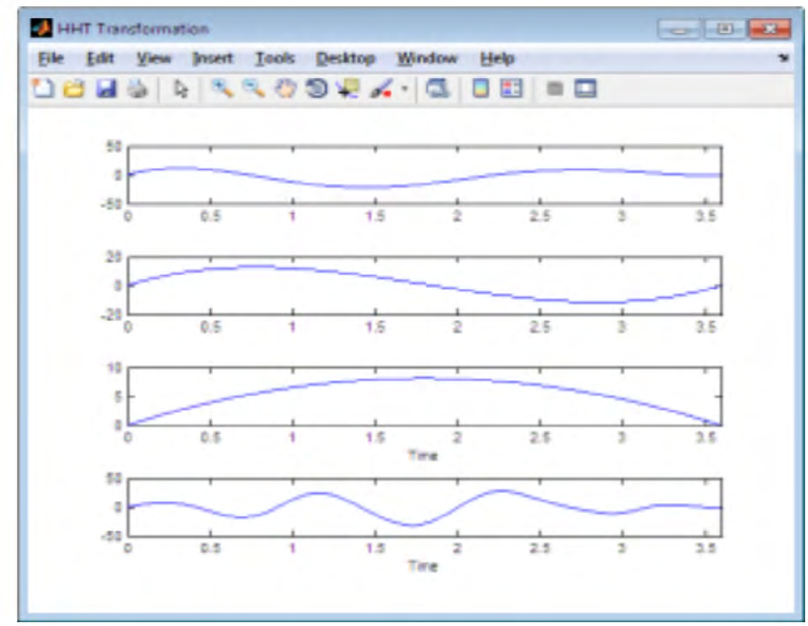

(e) 


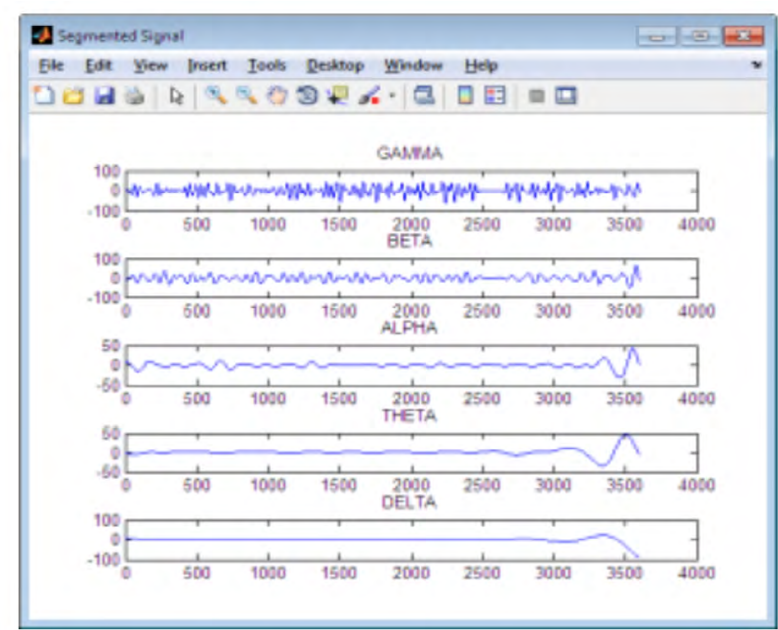

(f)

Figure 5. (e) Step input (f) Signal segmentation.

Source: own elaboration.

\subsection{FEATURE EXTRACTION}

Feature Extraction is obtained from the segmented parts of the EEG signal by the process of Signal segmentation. After completion of noise removal process in EEG signal, need to extract the feature parameters from the EEG signal. Statistical features are extracted they are Mean, Standard Deviation, Entropy, Skewness, Kurtosis.

Mean: In possibility, mean and expected value are used synonymously to consult one measure of the vital tendency either of a possibility distribution or of the random variable characterized via that distribution.

Standard Deviation: The Standard Deviation (SD) measures the amount of version or dispersion from the average. a low standard deviation indicates that the information points have a tendency to be very near the suggest (additionally known as expected fee); a high standard deviation suggests that the facts factors are unfold out over a big variety of values.

Entropy: Entropy is the common quantity or amount of information of statistics contained in each message acquired right here, message stands for an occasion, sample or person drawn from a distribution or information circulation entropy consequently characterizes our uncertainty about our source of records. 
Skewness: Skewness is a measure of the asymmetry of the opportunity of an actualvalued random variables approximately it suggests.

Kurtosis: Relative to a normal sharing Kurtosis is a measure of whether the data are pointed or level. If it has a particular top close to the average then it has data sets with high kurtosis, decline rather rapidly, if it tends to have a flat top near the mean rather than a sharp max out then the data sets have a low kurtosis. A uniform distribution would be the acute case.

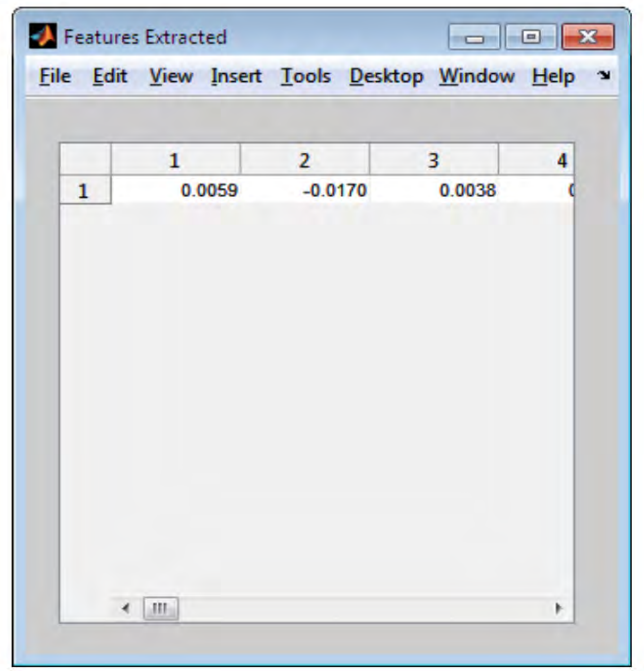

Figure 6. Feature Extraction.

Source: own elaboration.

\subsection{CLASSSIFICATION}

Based on the features extracted from the signals the classification process is carried out using SVM Classifier. The Support Vector Machine is an administered AI calculation that can be utilized to analyze information and perceive examples utilized for the procedure of characterization. The basic SVM takes a set of data as input and for each given input the SVM predicts, the two classes that forms the hyper plane, making it a non-probabilistic double straight classifier. For SVM set of training examples are carried out by marking that belongs to one or two categories, so that it builds a model for classification. 


\section{SVM Classifier:}

\section{The SVM algorithm}

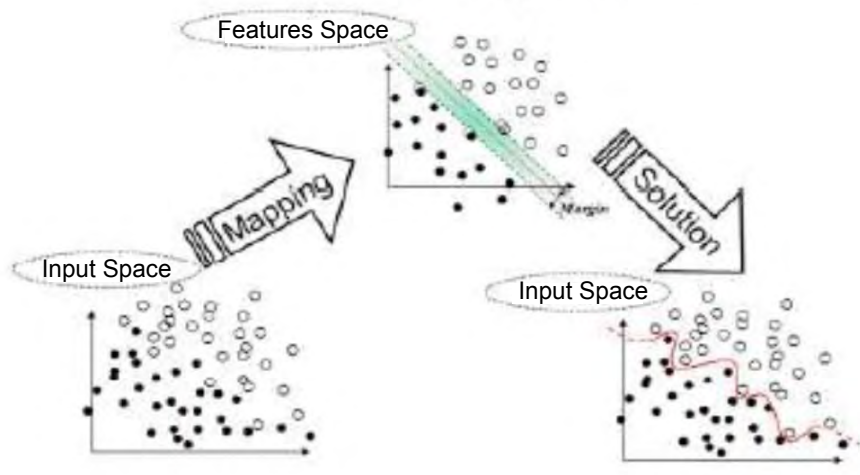

Figure 7. SVM algorithm.

Source: own elaboration.

SVM maps input vectors to a higher dimensional vector space where an ideal overexcited plane is built. Among the numerous hyper planes accessible, there is only one hyper plane that expands the separation among itself and the closest information vectors of every classification. This overexcited plane which amplifies the edge is known as the most ideal isolating overexcited plane and the edge is characterized as the total of separations of the hyper plane to the nearest preparing vectors of every class. For identifying the right hyper plane there are five types of situations.

Expression for hyper plane

$\mathrm{w} \cdot \mathrm{x}+\mathrm{b}=0$

$\mathrm{x}$ - Set of guidance vectors

$\mathrm{w}$ - Vectors perpendicular to the separating hyper plane

$\mathrm{b}$ - Offset parameter which allows the increase of the margin

The Support Vector Machine has the following advantages

- For clear margin of separation, the SVM really works well.

- $\quad$ For high dimensional spaces SVM is more effective. 
- In the event that the quantity of measurements is more noteworthy than the quantity of tests the SVM is more effective.

- It is memory productive why since it utilizes a subset of preparing focuses in the choice capacity called bolster vectors.

Figure 8. Classification.

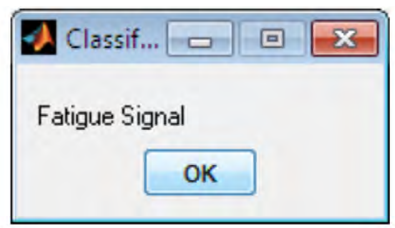

Source: own elaboration.

\section{RESULTS}

The performance analysis of the Support Vector Machine is analyzed, compared to the Neural Network Classifier the accuracy of the drowsiness identification is increased. This observation makes use of kernel capabilities which includes the gaussian radial foundation characteristic and the polynomial feature. SVM makes the process more simple and more efficient compared to neural network classifier.

\section{CONCLUSIONS}

From the research work that has been carried out with the machine learning technique we can identify that the best classification accuracy is achieved by the SVM classifier. WignerVille distribution (WVD) is employed to extract features from the EEG signals. The EMD technique decomposes a dataset into a finite and often small number of intrinsic mode functions (IMFs) were employed to segment the EEG signals. The benchmarked dataset that has been used in the existing works. A robust and computationally low-intensive waveletbased features have been used for the proposed EEG classification system.

The main advantage of the Support Vector Machine compared to other classifiers is that if you are working with an unbalanced data set then SVM is a good choice. In SVM the computational complexity is reduced and classification efficiency is increased compared to other classifiers mainly due to the global optimization functions included. The classifier has 
shown a terrific live performance in class. DWT presents an awesome nearby portrayal of the wavering added substances of the non-stationary or nonlinear sign. This system gives preferable sort exactness over a couple of methodologies contemplated beforehand.

\section{ACKNOWLEDGEMENT}

This work was supported by the department of Electronics and Instrumentation department of Kalasalingam Academy of Research and Education.

\section{REFERENCES}

Bajaj, V., \& Pachori, R. B. (2012) Separation of Rhythms of EEG Signals Based on Hilbert-Huang Transformation with Application to Seizure Detection. In: Lee, G., Howard, D., Kang, J. J., \& Ślęzak, D. (eds.), Convergence and Hybrid Information Technology. ICHIT 2012. Lecture Notes in Computer Science, vol. 7425. Springer, Berlin, Heidelberg. https://doi.org/10.1007/978-3-642-32645-5_62

Ghen, L.-I., Zhao, Y., Zhang, J., \& Zou, J.-Z. (2015). Automatic detection of alertness/ drowsiness from physiological signals using wavelet-based nonlinear features and machine learning. Expert Systems with Applications: An International Journal, 42(21), 73447355. https://dl.acm.org/doi/10.1016/j.eswa.2015.05.028

Gurudath, N., \& Riley, H. B. (2014). Drowsy Driving Detection by EEG Analysis Using Wavelet Transform and K-Means Clustering. Procedia Computer Science, 34, 400-409. https://doi.org/10.1016/j.procs.2014.07.045

Kar, S., Bhagat, M., \& Routray, A. (2010). EEG signal analysis for the assessment and quantification of driver's fatigue. Transportation Research Part F: Traffic Psychology and Behaviour, 13(5), 297-306, https://doi.org/10.1016/j.trf.2010.06.006

Kumar, G. R., Raju, S. V. P., \& Kumar, D. S. (2012). Classification of EEG signals for drowsiness Detection in brain and computer interface. GESJ: Computer Science and Telecommunications, 4(36). https://www.researchgate.net/publication/261100256_ Classification_of_EEG_Signals_for_Drowsiness_Detection_in_Brain_and_ Computer_Interface 
Majumdar, K. (2011). Human scalp EEG processing: Various soft computing approaches. Applied Soft Computing, 11(8), 4433-4447. https://doi.org/10.1016/j.asoc.2011.07.004

Mardi, Z., Ashtiani, S. N. M., \& Mikaili, M. (2011). EEG-Based Drowsiness Detection for Safe Driving Using Chaotic Features and Statistical Tests. Fournal of Medical Signals E Sensors, 1(2), 130-137. https://pubmed.ncbi.nlm.nih.gov/22606668/

Picot, A., Charbonnier, S., \& Caplier, A. (2011). EOG-based drowsiness detection: Comparison between a fuzzy system and two supervised learning classifiers. IFAC Proceedings Volumes, 44(1), 14283-14288. https://doi.org/10.3182/20110828-6IT-1002.00706

Picot, A., Gharbonnier, S., \& Caplier, A. (2012). On-Line Detection of Drowsiness using Brain and Visual Information. IEEE Transactions on Systems, Man, and Cybernetics - Part A: Systems and Humans, 42(3), 1083-4427. https://ieeexplore.ieee.org/ document/6029307

Zhang, G., Wang, H., \& Fu, R. (2014). Automated Detection of Driver Fatigue Based on Entropy and Complexity Measures. IEEE transactions on intelligent transportation systems, 15(1). https://ieeexplore.ieee.org/document/6589164 
